\title{
Retenção do lítio em solos de áreas adjacentes às impactadas pelo rompimento da barragem do Fundão, Mariana - MG
}

\author{
Lithium retention in soils in areas adjacent to those impacted by the Fundão dam rupture, Mariana \\ - MG
}

Retención de litio en suelos en áreas adyacentes a las impactadas por el colapso de la represa

Fundão, Mariana - MG

Recebido: 26/08/2021 | Revisado: 01/09/2021 | Aceito: 03/09/2021 | Publicado: 06/09/2021

\author{
Roger Levy \\ ORCID: https://orcid.org/0000-0002-9923-3925 \\ Universidade Vale do Rio Verde, Brasil \\ E-mail: roglevy.ambiental@yahoo.com \\ Fernanda Leal Miranda \\ ORCID: https://orcid.org/0000-0003-1488-4559 \\ Universidade Vale do Rio Verde, Brasil \\ E-mail: nanda454@ hotmail.com \\ Fernando Soares Firmino \\ ORCID: https://orcid.org/0000-0002-5241-8615 \\ Universidade Vale do Rio Verde, Brasil \\ E-mail: fsf.seguranca@gmail.com \\ Rosângela Francisca de Paula Vitor Marques \\ ORCID: https://orcid.org/0000-0001-6646-0809 \\ Universidade Vale do Rio Verde, Brasil \\ E-mail: roeflorestal@hotmail.com \\ Michael Silveira Thebaldi \\ ORCID: https://orcid.org/0000-0002-4579-6714 \\ Universidade Federal de Lavras, Brasil \\ E-mail: michael.thebaldi@ufla.br \\ Marcelo Henrique Fernandes de Faria Rocha \\ ORCID: https://orcid.org/0000-0001-6041-6233 \\ Universidade Vale do Rio Verde, Brasil \\ E-mail: mhffr@yahoo.com \\ Alisson Souza de Oliveira \\ ORCID: https://orcid.org/0000-0001-7885-9542 \\ Universidade Vale do Rio Verde, Brasil \\ E-mail: alissonso@hotmail.com \\ Claudiomir da Silva dos Santos \\ ORCID: https://orcid.org/0000-0002-0007-7273 \\ Instituto Federal do Sul de Minas, Brasil \\ E-mail:claudiomirsilvasantos@gmail.com \\ Fabrício dos Santos Ritá \\ ORCID: https://orcid.org/0000-0003-2009-3673 \\ Instituto Federal Sul de Minas, Brasil \\ E-mailfabriciosantosrita@gmail.com \\ Karina de Jesus Soares \\ ORCID: https://orcid.org/0000-0002-0504-0082 \\ Universidade Estadual do Norte Fluminense, Brasil \\ E-mail:kjsoares.agro@gmail.com
}

\begin{abstract}
Resumo
Objetivou-se analisar a sorção de Lítio (Li) em solos não contaminados de áreas adjacentes à que recebeu rejeito da Barragem de Fundão, por meio de modelos matemáticos e tempo de contato da solução e solo, visando identificar a potencialidade de contaminação do solo e águas subterrâneas. Coletaram-se amostras deformadas de solo, na profundidade de 0 - $20 \mathrm{~cm}$ para caraterização química. Posteriormente pesou-se terra fina seca ao ar e colocou-se em béqueres com $20 \mathrm{~mL}$ de cloreto de lítio. As concentrações utilizadas foram baseadas na legislação vigente, para classe 1, sendo: 2,3; 5,2; 10,1; 20,3; 30,1 e 50,0 $\mathrm{mg} \mathrm{L}^{-1}$ de Li. Para identificação do melhor tempo de contato entre solução e adsorvente, a solução permaneceu em repouso (1, 12 e 24 horas), à temperatura constante de $20{ }^{\circ} \mathrm{C}$. Os parâmetros das isotermas Linear, Langmuir e Freundlich foram ajustados objetivando obter curvas experimentais da solução. Assim utilizou-se o coeficiente de determinação (R2) e teste F. O solo apresenta baixa capacidade de adsorção devido às
\end{abstract}


características químicas, portanto, vulnerável à contaminação das águas subterrâneas. A maior adsorção foi no tempo de 24 horas e o modelo com melhor ajuste aos dados foi o de Freundlich.

Palavras-chave: Adsorção; Isoterma de sorção; Poluição do solo e águas subterrâneas.

\begin{abstract}
The objective of this study was to analyze a sorption of Lithium (Li) in uncontaminated soils from areas adjacent to the tailings of the Fundão Dam, through mathematical models and contact time of the solution and soil, to identify the potential for contamination of the soil and groundwater. Deformed soil samples were collected at a depth of $0-20 \mathrm{~cm}$ for chemical characterization. Afterwards, air-dried fine earth was weighed and placed in beakers with $20 \mathrm{ml}$ of lithium chloride. Those used were based on current legislation, for class 1 , as follows: $2.3 ; 5.2 ; 10.1 ; 20.3 ; 30.1$ and $50.0 \mathrm{mg} \mathrm{L}^{-1}$ of Li. To identify the best contact time between solution and adsorbent, a solution remained at rest $(1,12$ and 24 hours) at a constant temperature of $20{ }^{\circ} \mathrm{C}$. Linear, Langmuir and Freundlich isotherms were fitted to obtain experimental curves of the solution. Thus, it uses the coefficient of determination (R2) and the F test. The soil has a low adsorption capacity due to its chemical characteristics, therefore, it is vulnerable to groundwater contamination. The greatest adsorption was within 24 hours and the model with the best fit to the data was Freundlich.
\end{abstract}

Keywords: Adsorption; Sorption isotherm; Soil and groundwater pollution.

\title{
Resumen
}

El objetivo fue analizar la sorción de Litio (Li) en suelos no contaminados de áreas adyacentes a la que recibió relaves de la Represa Fundão, a través de modelos matemáticos y tiempo de contacto de la solución y suelo, con el fin de identificar el potencial de contaminación de el suelo y las aguas subterráneas. Se recolectaron muestras de suelo deformado a una profundidad de 0 a $20 \mathrm{~cm}$ para la caracterización química. Posteriormente, se pesó tierra fina secada al aire y se colocó en vasos de precipitados con $20 \mathrm{ml}$ de cloruro de litio. Las concentraciones utilizadas se basaron en la legislación vigente, para la clase 1 , de la siguiente manera: 2.3; 5,2; 10,1; 20,3; 30.1 y $50.0 \mathrm{mg} \mathrm{L}^{-1}$ de Li Para identificar el mejor tiempo de contacto entre solución y adsorbente, la solución permaneció en reposo (1, 12 y 24 horas) a una temperatura constante de $20^{\circ}$ C. Se ajustaron los parámetros de las isotermas Lineal, Langmuir y Freundlich para obtener curvas experimentales de la solución. Así, se utilizó el coeficiente de determinación (R2) y la prueba F. El suelo tiene una baja capacidad de adsorción debido a sus características químicas, por lo que es vulnerable a la contaminación de las aguas subterráneas. La adsorción más alta se produjo dentro de las 24 horas y el modelo que mejor se ajustó a los datos fue el modelo de Freundlich.

Palabras clave: Adsorción; Isoterma de sorción; Contaminación del suelo y las aguas subterrâneas.

\section{Introdução}

Inobstante a mineração industrial ser componente salutar da cadeia socioeconômica do Estado de Minas Gerais, especialmente do Quadrilátero Ferrífero, região na qual tal atividade tem notável presença desde os longevos tempos do ciclo do ouro (Rocha et. al., 2021), os processos de exploração e beneficiamento mineral, quando ancorados em concepções de engenharia reconhecidamente obsoletas, podem desencadear eventos catastróficos como os rompimentos de barragens dedicadas à contenção de rejeitos minerários.

Dentre a miríade de fatores que podem culminar no rompimento de barragens de rejeitos, dois são preponderantes: o abalo da estrutura em função da ocorrência de fenômenos sísmicos e/ou mesmo o planejamento errático do maciço. Em 05/11/2015, o colapso da barragem de Fundão, localizada no município de Mariana, estado de Minas Gerais, construída especificamente para acumulação de resíduos oriundos à mineração de ferro, liberou uma avalanche de lama residual e causou um desastre ambiental sem precedentes na história do Brasil (Lopes, 2016). De acordo com o Instituto Brasileiro de Meio ambiente (IBAMA), além do severo impacto socioambiental, do qual derivam intermináveis imbróglios judiciais, os impactos diretos sobre a fauna e a flora existentes em toda extensão da bacia hidrográfica do Rio Doce assim como sobre a qualidade das águas do curso hídrico em epígrafe foram devastadores (Brasil, 2015).

Os monitoramentos emergenciais empreendidos pelo Instituto Mineiro de Gestão das Águas (IGAM) identificaram concentrações alarmantes de manganês, alumínio dissolvido e ferro dissolvido nas amostras de águas superficiais em vários pontos do Rio Doce. Os baixos teores de argila e matéria orgânica detectados nas diversas amostragens de solo apontam para uma diminuição da capacidade de troca catiônica do mesmo, o que certamente acarretará baixa fertilidade. (Minas Gerais, 2016). 
Os níveis de elementos traço, por sua vez, apresentam valores inferiores aos adotados como referência para avaliação da contaminação de metais no solo segundo a Resolução CONAMA 420/2009 (Brasil, 2009).

Segundo Zuquette et. al (2008), os processos físicos, químicos e biológicos influenciam diretamente a capacidade de acumulação ou mobilidade de diversos elementos no solo e os fatores que controlam o destino final dependerão das condições geológicas, climáticas, físico-químicas e biológicas (Bini et al., 2011; Haddad et al., 2013). Os principais aspectos químicos que influenciam a capacidade de acumulação ou mobilidade são a concentração da solução no solo, o pH, a capacidade de troca de cátions, as reações de dissolução e/ou precipitação e as trocas iônicas entre os nutrientes que estão na solução com aqueles da fase sólida durante o processo de transporte (Bolzani; Oliveira; Lautenschlager, 2012). O solo possui grande capacidade de retenção dos elementos de baixa concentração. Entretanto, se esta capacidade for ultrapassada, os elementos traço alterarão sua disponibilidade para o meio (Oliveira et al., 2010). Desta feita, para reduzir o risco potencial de contaminação por compostos nocivos, provenientes da lama e rejeitos ao meio, é necessário conhecer as interações com o solo (Corrêa et al., 2008).

Um aspecto fundamental na determinação das quantidades de elementos traços nos solos, como o Lítio (Li), refere-se à sua movimentação no perfil, que depende das propriedades físico-químicas, físicas e químicas de cada tipo de solo (Campos, 2010). Nesse sentido, existem estudos de sorção e de mobilidade dos solutos no solo a partir da aplicação de métodos de batelada (Nicochelli et al., 2012). Segundo Freitas (2010), cada solo possui uma capacidade adsortiva própria sendo que o conhecimento desta capacidade oferecerá subsídios para a previsão de fitotoxicidade e possível contaminação do lençol freático. Assim, a utilização de isotermas de adsorção para estudos dos fenômenos de adsorção é uma boa maneira de estudar o comportamento de um metal no solo é aplicando modelos físico-químicos de adsorção.

Entre os diversos modelos que descrevem a adsorção dos elementos no solo, os mais citados são os propostos por Langmuir e por Freundlich. Esses modelos são facilmente aplicáveis para adsorção de íons pelo solo e tem se apresentado como adequados para os estudos de adsorção de alguns metais em solos brasileiros (Freitas, 2010).

Neste sentido, objetivou-se analisar a adsorção do lítio em um solo de Mariana não contaminado pela lama de rejeito proveniente da tragédia de fundão através de ensaios de sorção dos modelos matemáticos Linear, Langmuir e Freundlich e tempo de contato da solução e o solo, visando identificar a potencialidade de contaminação do solo e das águas subterrâneas em função dos resultados obtidos.

\section{Metodologia}

A pesquisa do presente estudo segundo Zanella (2013) possui uma abordagem quantitativa, sendo a pesquisa do tipo causal e método utilizado para o presente estudo é o experimental.

Assim, amostras simples e deformadas de solo foram coletadas pelo Departamento de Ciências Florestais da Universidade Federal de Lavras - UFLA com auxílio de um trado holandês na camada de $0-20 \mathrm{~cm}$, no município de Mariana em uma propriedade particular próxima ao local em que ocorreu o acúmulo de lama, porém em uma área não contaminada pelo rejeito, as quais apresentam as características do solo original.

Segundo o estudo de impacto ambiental da empresa Samarco Mineração, na unidade predomina a associação de latossolo e cambissolo, sendo que na área do córrego do Fundão há o predomínio de cambissolo háplico. A unidade representada pelos cambissolos, corresponde a solos identificados principalmente nos sopés das escarpas, sendo constituídos por material mineral, com horizonte B incipiente (Bi) subjacente a qualquer tipo de horizonte superficial (Embrapa, 2013). Constituem solos em estágio intermediário de evolução, isto é, que não sofreram alterações físicas e químicas muito avançadas. São solos, em geral, não muito profundos, que apresentam teores relativamente elevados de minerais primários facilmente intemperizáveis, atividade da fração argila de média a alta e solo com discreta variação de textura. Devido ao seu 
grau intermediário de evolução, ocorrem principalmente em médias vertentes, associadas à Escarpa Oriental do Caraça (unidade geomorfológica) com declividades mais acentuadas, sob influência principalmente de litologias da Formação Moeda (quartizitos) e também da Formação Cauê (itabiritos).

A caracterização química do solo por meio de amostra composta foi realizada no Laboratório de Ciências do Solo de acordo com metodologia proposta por EMBRAPA (2011) e fornecida pelo Departamento de Ciências Florestais da UFLA para o presente estudo. O solo é caracterizado como de textura argilosa sendo a análise granulométrica de 56,0\% de argila, 13,0\% de silte e 31,0\% de areia e suas características químicas apresentadas na Tabela 1.

Tabela 1. Características químicas do solo do município de Mariana em área adjacente ao acúmulo de lama proveniente de rompimento de barragem de rejeito de mineração de ferro.

\begin{tabular}{ccc}
\hline Parâmetro & Valor & Unidade \\
\hline $\mathrm{pH}$ & 4,9 & - \\
$\mathrm{K}$ & 7,43 & $\mathrm{mg} \mathrm{dm}^{-3}$ \\
$\mathrm{P}$ & 3,72 & $\mathrm{mg} \mathrm{dm}^{-3}$ \\
$\mathrm{Na}$ & 7,38 & $\mathrm{mg} \mathrm{dm}^{-3}$ \\
$\mathrm{Ca}$ & 0,22 & $\mathrm{cmolc} \mathrm{dm}^{-3}$ \\
$\mathrm{Mg}$ & 0,10 & $\mathrm{cmolc} \mathrm{dm}^{-3}$ \\
$\mathrm{Al}$ & 0,20 & $\mathrm{cmolc} \mathrm{dm}^{-3}$ \\
$\mathrm{H}+\mathrm{Al}$ & 2,49 & $\mathrm{cmolc} \mathrm{dm}^{-3}$ \\
$\mathrm{t}$ & 0,54 & $\mathrm{cmolc} \mathrm{dm}^{-3}$ \\
$\mathrm{~T}$ & 2,83 & $\mathrm{cmolc} \mathrm{dm}^{-3}$ \\
$\mathrm{M} . \mathrm{O}$ & 0,63 & $\mathrm{dag} \mathrm{kg}^{-1}$ \\
$\mathrm{Zn}$ & 0,91 & $\mathrm{mg} \mathrm{dm}^{-3}$ \\
$\mathrm{Fe}$ & 45,72 & $\mathrm{mg} \mathrm{dm}^{-3}$ \\
$\mathrm{Mn}$ & 3,90 & $\mathrm{mg} \mathrm{dm}^{-3}$ \\
$\mathrm{Cu}$ & 39,99 & $\mathrm{mg} \mathrm{dm}^{-3}$ \\
$\mathrm{~B}$ & 0,50 & $\mathrm{mg} \mathrm{dm}^{-3}$ \\
$\mathrm{~S}$ & 24,03 & $\mathrm{mg} \mathrm{dm}^{-3}$ \\
\hline
\end{tabular}

Legenda: pH em águaP- Na - K- Fe - Zn- Mn- Cu- Extrador Mehlich 1; Ca - Mg- Al- Extrator: $\mathrm{KCl}-1$ mol/L $\mathrm{H}+$ Al- Extrator: SMP; $\mathrm{SB}=$ Soma de Bases Trocáveis CTC (t) - Capacidade de Troca Catiônica Efetiva; CTC (T) - Capacidade de Troca Catiônica a pH 7,0; Mat. Org. (MO) - Oxidação: Na2Cr2O7 4N+ H2SO4 $10 \mathrm{~N}$;

Fonte: DCS - UFLA, cedido pelo DCF 2018 (adaptado).

O experimento foi realizado com as amostras de solo destorroadas e passadas através de uma peneira de malha de 2,00 mm de abertura. Posteriormente, o solo foi seco ao ar, obtendo terra fina seca ao ar (TFSA) no Laboratório de Solos do Departamento de Recursos Hídricos e Saneamento da Universidade Federal de Lavras (UFLA). Para a construção da curva de calibração, utilizaram-se concentrações de lítio conhecidas $\left(2,3 ; 5,2 ; 10,1 ; 20,3 ; 30,1\right.$ e $\left.50,0 \mathrm{mg} \mathrm{L}^{-1}\right)$ e realizado a leitura no fotômetro de chama modelo B462 da Micronal. Posteriormente plotou-se os dados no excel e ajustou-se uma curva de calibração por meio de regressão sendo utilizado o modelo exponencial.

Para a realização do experimento pesou-se $10 \mathrm{~g}$ de Terra Fina Seca ao ar (TFSA) em balança de precisão que foi colocado em béqueres em triplicata e adicionou-se $20 \mathrm{~mL}$ de soluções preparadas de cloreto de lítio (Li) com concentrações de 2,3; 5,2;10,1;20,3; 30,1 e 50,0 mg L-1 de Li, o qual foram baseadas a partir das concentrações permitidas pela resolução CONAMA n ${ }^{\circ} 357$ de 2005 (BRASIL, 2005), para água doce Classe 1.

A análise das isotermas de sorção e as curvas obtidas a partir de ensaios de equilíbrio em lote que, por sua vez, consistem na agitação de misturas compostas por uma porção de solo e uma solução contendo um contaminante com concentração inicial conhecida, com a finalidade de aumentar o contato da solução com o solo, os béqueres contendo as amostras foram agitados por um período de 24 horas. Após a agitação, as amostras foram acondicionadas em estufa incubadora de BOD (Demanda Bioquímica de Oxigênio) e mantidas nesta por períodos de 1 hora, 12 horas, e 24 horas, a uma temperatura 
de $20^{\circ} \mathrm{C}$.

Para determinação da concentração de equilíbrio da solução, fez-se a filtragem da solução em papel filtro e coletou-se amostras de $10 \mathrm{~mL}$ de sobrenadante. Assim, a adsorção de Li ao solo pôde ser calculada por meio da Equação 1.

$$
S=\left(C_{i}-C_{e}\right) \times \frac{V}{m}
$$

Em que:

S - adsorção de Li pelo solo ( $\left.\mathrm{mg} \mathrm{kg}^{-1}\right)$;

$\mathrm{Ci}$ - concentração inicial de Li nas soluções $\left(\mathrm{mg} \mathrm{L}^{-1}\right)$

$\mathrm{Ce}$ - concentração de equilíbrio de Li nas soluções $\left(\mathrm{mg} \mathrm{L}^{-1}\right)$;

$\mathrm{m}$ - massa da TFSE $(\mathrm{kg})$;

$\mathrm{V}$ - volume da solução adicionada a cada um dos béqueres (L).

Com a obtenção da adsorção em cada tratamento para suas respectivas concentrações de equilíbrio, os parâmetros dos modelos matemáticos de isotermas Linear, de Langmuir e de Freundlich foram ajustados. Os modelos matemáticos das isotermas Linear, de Langmuir e de Freundlich são mostrados nas Equações 2, 3 e 4, respectivamente.

$$
\begin{aligned}
& S=K_{d} \times C_{e} \\
& S=\frac{m_{L} \times C_{w} \times b}{1+\left(K_{L} \times C_{E}\right)} \\
& S=K_{F} \times C_{e}^{\frac{1}{n}}
\end{aligned}
$$

Em que:

$\mathrm{Kd}$ - Constante relacionada à energia de ligação soluto-superfície adsorvente $\left(\mathrm{L} \mathrm{kg}^{-1}\right)$.

$\mathrm{KL}$ - Constante relacionada à energia de ligação soluto-superfície adsorvente $\left(\mathrm{L} \mathrm{mg}^{-1}\right)$;

b - Quantidade máxima de soluto que pode ser adsorvida $\left(\mathrm{mg} \mathrm{kg}^{-1}\right)$;

$\mathrm{KF}$ - Constante de capacidade de adsorção de Freundlich $\left(\mathrm{L} \mathrm{mg}^{-1}\right)$; e

$1 / \mathrm{n}$ - Constante relacionada à heterogeneidade da superfície.

A qualidade dos ajustes dos modelos matemáticos das isotermas foi avaliada pela determinação dos parâmetros estatísticos erro absoluto médio, raiz quadrado do erro médio, do coeficiente de determinação (R2) e pelo teste $\mathrm{F}$ a $5 \%$ de probabilidade.

\section{Resultados e Discussão}

Na Tabela 2 são apresentados os resultados do teste $\mathrm{F}$ a 5\% de probabilidade. Observou-se que o modelo matemático de isotermas de adsorção que apresentou melhor aderência aos dados observados foi o de Freundlich, sendo o ajuste significativo para os tempos de contato de 1 hora e 24 horas. Para o tempo de 12 horas nenhum ajuste foi significativo a $5 \%$ de probabilidade, porém, obteve-se para 12 horas de contato entre solução e adsorvente maior valor de F calculado para o ajuste obtido com o uso do modelo de Freundlich.

Corroborando com o obtido para o teste $\mathrm{F}$ (Tabela 2), foram verificados maiores valores de $\mathrm{R}^{2}$ para o ajuste do modelo de Freundlich aos dados observados de adsorção de Li ao solo de áreas adjacentes às áreas impactadas pelo rompimento da barragem do Fundão, em Mariana, para todos os tempos de contato avaliados (Tabela 3), porém ressalta-se que as condições de equilíbrio são geralmente atingidas em 24 horas, conformo citado por USEPA (1992), sendo que o tempo de equilíbrio deve ser o mínimo necessário para estabelecer uma taxa de variação da concentração do soluto em solução, igual ou menor que $5 \%$ por um intervalo de $24 \mathrm{~h}$. 
Tabela 2. Resultados do Teste F aplicados aos modelos matemáticos Linear, Langmuir e Freundlich na avaliação da adsorção de lítio (Li) ao solo de área impactada pelo rompimento da barragem do Fundão, Mariana - MG, Brasil.

\begin{tabular}{ccccc}
\hline \multirow{2}{*}{ Tempo } & \multicolumn{3}{c}{ Isotermas de adsorção } & \multirow{2}{*}{ F teórico } \\
\cline { 2 - 4 } & Linear & Langmuir & Freundlich & \\
\hline 1 hora & 4,08184 & 3,99203 & $235,8503^{*}$ & \multirow{2}{*}{6,607891} \\
12 horas & 2,216771 & 2,216809 & 3,937454 & \\
24 horas & 2,048582 & 2,047652 & $9,190998^{*}$ & \\
\hline
\end{tabular}

Valores seguidos de asterisco são significativos ao teste de $\mathrm{F}$ a $5 \%$ de probabilidade estatística. Fonte: Autores (2018).

Os parâmetros de ajuste dos modelos matemáticos de isotermas de adsorção Linear, de Langmuir e de Freundlich são mostrados na Tabela 3, juntamente com os coeficientes de determinação obtidos para os ajustes.

Tabela 3. Coeficiente de determinação $\left(\mathrm{R}^{2}\right)$ e parâmetros das isotermas Linear, de Langmuir e Freundlich para adsorção de Li a solo de área impactadas pelo rompimento da barragem do Fundão, Mariana - MG.

\begin{tabular}{|c|c|c|c|c|c|c|}
\hline \multicolumn{3}{|c|}{ Contato de 1 hora } & \multicolumn{2}{|c|}{ Contato de 12 horas } & \multicolumn{2}{|c|}{ Contato de 24 horas } \\
\hline Modelo & Parâmetros & $\mathrm{R}^{2}$ & Parâmetros & $\mathrm{R}^{2}$ & Parâmetros & $\mathrm{R}^{2}$ \\
\hline Linear & $\mathrm{Kd}=9,4339$ & 0,75 & $\mathrm{Kd}=11,4886$ & 0,49 & $\mathrm{Kd}=12,3366$ & 0,47 \\
\hline Langmuir & $\begin{array}{c}\mathrm{K}=0,00093 \\
\mathrm{~B}=10054,182\end{array}$ & 0,74 & $\begin{array}{c}\mathrm{K}_{\mathrm{l}}=0,000054 \\
\mathrm{~B}=213950,609\end{array}$ & 0,49 & $\begin{array}{c}\mathrm{K}_{\mathrm{l}}=0,00051047 \\
\mathrm{~B}=24231,533\end{array}$ & 0,46 \\
\hline Freundlich & $\begin{array}{l}\mathrm{K}_{\mathrm{f}}=0,05159 \\
\mathrm{n}=0,238186\end{array}$ & 0,99 & $\begin{array}{c}\mathrm{K}_{\mathrm{F}}=0,70992 \\
\mathrm{n}=0,315803\end{array}$ & 0,64 & $\begin{array}{l}\mathrm{K}_{\mathrm{F}}=0,01851 \\
\mathrm{n}=0,156977\end{array}$ & 0,82 \\
\hline
\end{tabular}

Fonte: Autores (2018).

As isotermas ajustadas para os modelos Linear, de Langmuir e de Freundlich para os tempos de 1, 12 e 24 horas, podem ser vistas na Figura 1 (A, B e C).

De acordo com Site (2001), o parâmetro " $n$ ” indica os diferentes níveis de energia da interação do adsorvente pelo adsorvato e que, para valores compreendidos entre de 1 a 10, a adsorção é considerada favorável. Neste trabalho os valores obtidos deste parâmetro foram inferiores a 1, o que indica uma adsorção desfavorável. Essa tendência a uma adsorção desfavorável de Li ao solo, para todos os tempos de contato pode ser constatada ao se observar a Figura 1 (A, B e C), em que para certos incrementos da concentração de equilíbrio de Li na solução, há incrementos proporcionalmente inferiores de adsorção. 
Figura 1. Modelos matemáticos de adsorção Linear, de Langmuir e de Freundlich de Li ao solo adjacente de área impactada pelo rompimento da barragem do Fundão, Mariana - MG, para tempos de contato de 1 hora (A), 12 horas (B) e 24 horas $(\mathrm{C})$.

$1 \mathrm{~A}$

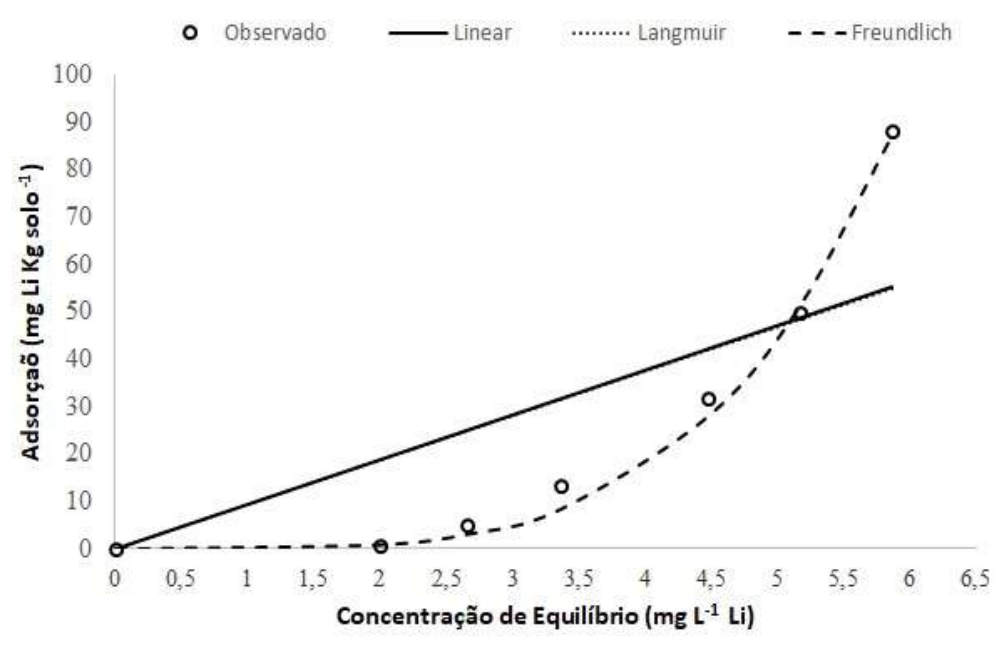

$1 \mathrm{~B}$

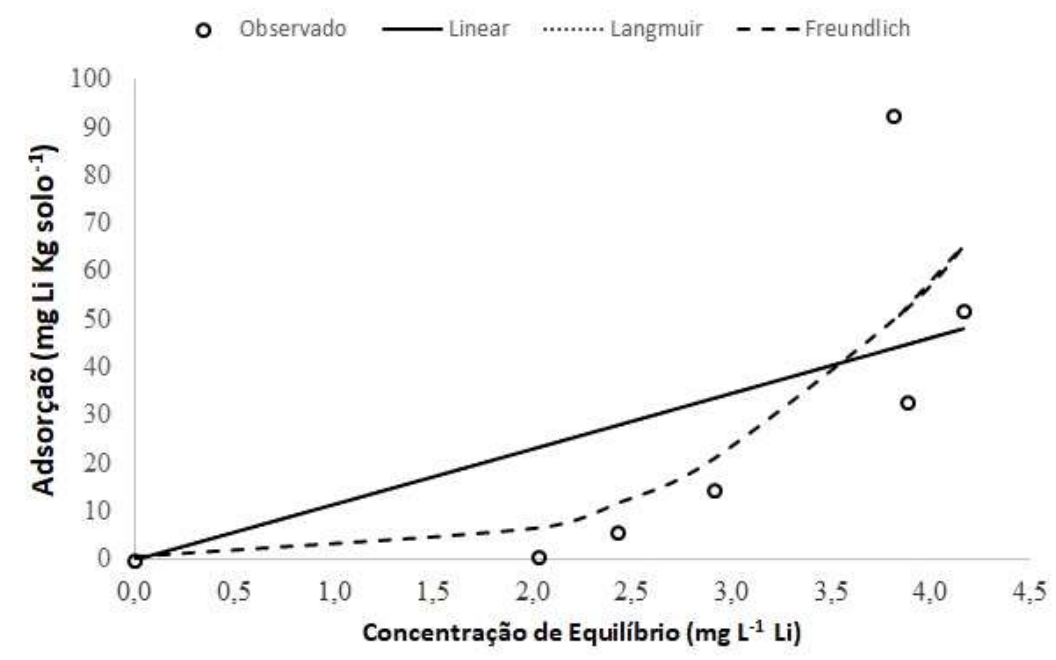

$1 \mathrm{C}$

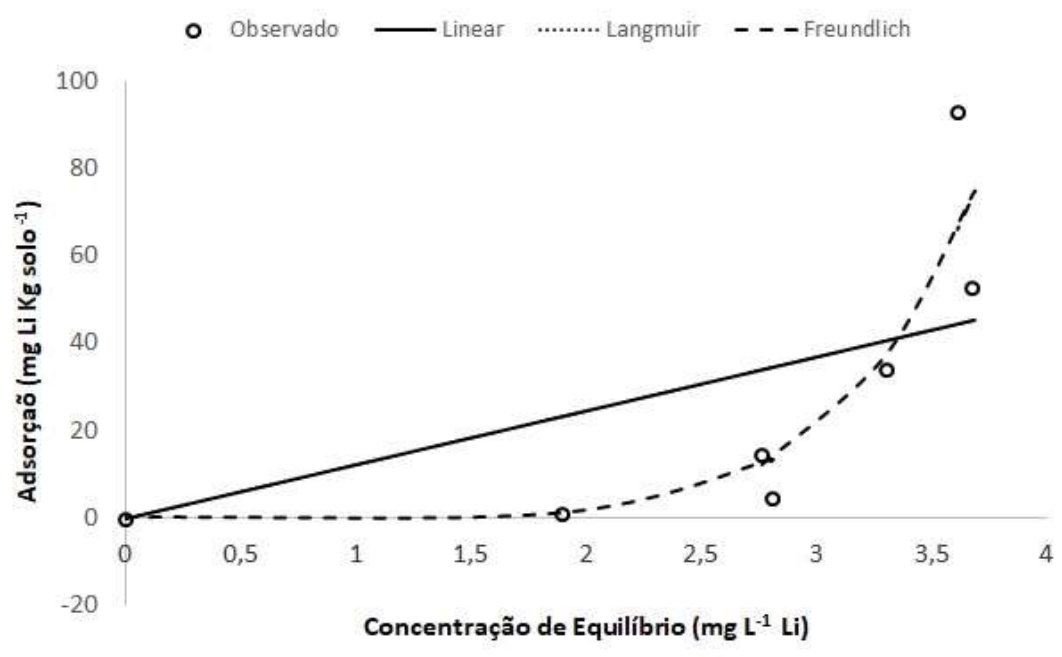

Fonte: Autores (2018). 
Como exemplo, para concentrações de equilíbrio próximas a valores entre 2 e $3 \mathrm{mg} \mathrm{L}^{-1}$ não se teve adsorção superior a aproximadamente $10 \mathrm{mg} \mathrm{kg}^{-1}$. Após isso, para pequenos incrementos de concentração de equilíbrio (Ce), observa-se um aumento substancial da adsorção, que chega a valores simulados de até $90 \mathrm{mg} \cdot \mathrm{kg}^{-1}$ para o tempo de contato de 1 hora (Figura 1A). Por outro lado, o valor de $1 / \mathrm{n}$ foi maior do $\mathrm{n}$, indicando que o adsorvente possui maior afinidade pelo solvente, sugerindo que há uma forte atração intermolecular entre o adsorvato (concentração de lítio) e o adsorvente (solo) (Delle-Site, 2001).

Avaliando-se a Tabela 1, observou-se valores baixos de $\mathrm{pH}$ e, em decorrência do processo de contaminação, estes podem diminuir ainda mais, já que a entrada de um cátion divalente nos sítios de troca do solo corresponde à saída de 1 ou 2 íons $\mathrm{H}+$. Deve-se ressaltar que nem todas as moléculas presentes no fluido podem ser adsorvidas quimicamente, somente aquelas capazes de se ligar ao sítio ativo (Nascimento et. al, 2014).

Solos mais intemperizados apresentam maiores valores de Ponto de Carga Zero - PCZ, assim sendo, possuem tendência ao caráter mais eletropositivo (Baldotto; Velloso, 2014), o que acarreta nestes menor capacidade de troca catiônica (CTC), havendo maior tendência à adsorção de ânions. Assim, quanto menor o pH, menor será a capacidade de retenção de metais (Maguirre et al. 1981).

Oliveira et al. (2013) afirmam que o solo com pH inferior, ou seja, solos ácidos, tem capacidade de troca catiônica baixa, propiciando uma menor interação de íons positivos com o solo. Em relação à CTC, o solo apresenta baixa CTC e, segundo Corrêa et al. (2008), em ambientes ácidos, o pH é menor que o Ponto de carga zero, assim, consequentemente, a superfície dos sítios ativos torna-se positivamente carregada quando se associa com os prótons provenientes da solução, sob condições ácidas. Assim sendo, há uma tendência para que os cátions presentes concorram com o Lítio quanto a sua adsorção, aumentando a disponibilidade do contaminante no solo, fazendo que o mesmo seja lixiviado para as camadas mais profundas, bem como outros cátions, quando há presença de fluxo de água.

A teoria que sustenta a técnica de determinação do ponto de carga zero assume que os prótons $\mathrm{H}^{+}$e os grupamentos hidroxílicos $\mathrm{OH}^{-}$constituem íons determinantes em potencial. O adsorvente em solução aquosa pode adsorver íons $\mathrm{OH}^{-} \mathrm{ou} \mathrm{H}^{+}$, assim, a carga superficial de cada partícula dependerá do pH da solução. Desta forma, os grupamentos superficiais de cada sítio ativo do adsorvente podem dissociar ou associar prótons da solução, dependendo das propriedades do adsorvente e do $\mathrm{pH}$ da solução. Consequentemente, a superfície dos sítios ativos torna-se positivamente carregada quando se associa com os prótons provenientes da solução, sob condições ácidas, ou negativamente carregadas quando ocorre a perda de prótons para a solução, sob condições alcalinas (Cerovic et al., 2007).

Em certas condições químicas e físicas, a adsorção provém de um equilíbrio entre forças presentes no adsorvente e a energia cinética do adsorvato. Quando isso acontece, a superfície do adsorvente fica coberta com um mesmo íon ou substância, que se torna fonte de atração para a mesma substância ou íon, formando-se camadas múltiplas (Schmal, 2010). Esse é o caso da isoterma não-favorável de Freundlich - a faixa de Ce em que há um grande aumento da adsorção, pode ser explicada pela atração mútua de mesmas espécies químicas, formando-se várias camadas.

Assim sendo, a partir de uma certa concentração superior do adsorvato, este já possui energia suficiente, fazendo que a adsorção ocorra, sendo esta, mais rápida para maiores concentrações iniciais e facilitando a ocorrência de adsorções subsequentes (Vilela et al, 2018).

Cabe ressalvar, de acordo Nicochellie et al. (2012) e Yamamura (2009), a isoterma estimada pelo modelo de Freundlich supõe sorção ilimitada, isto é, o solo nunca é saturado para atingir sua capacidade de retenção, pois o número de sítios de adsorção é muito maior do que a quantidade de moléculas de soluto, portanto, a isoterma não exibe um patamar de concentração máxima, como é apresentado para a isoterma de Langmuir. Estas informações corroboram com as afirmações de Cooney (1999), o qual afirma que o modelo de Freundlich não impõe qualquer exigência de que a cobertura deve se aproximar 
de um valor constante, porém, de acordo com o processo de adsorção, fisicamente é impossível, ou seja, dados experimentais que possuem elevados valores de Ce não são bem ajustados à equação de Freundlich.

Salienta-se que para este estudo, não foram observadas altas concentrações de equilíbrio e que, quanto maior estas fossem, se teria valores similares de adsorção, o que mostraria a saturação ou completa ocupação dos sítios de troca por íons. Assim sendo, na prática não há indicação que a isoterma de Freundlich é a que melhor representa a adsorção de Li a solos com esta característica e sim, que, para as condições estabelecidas para este estudo, a adsorção máxima não foi obtida, mesmo com a baixa CTC do solo, sendo então claramente determinado que a modelagem e ajuste de parâmetros de isotermas aqui apresentados são válidos apenas para concentrações iniciais de Li em solução de até $50 \mathrm{mg} \mathrm{L}^{-1}$.

Diversos trabalhos foram realizados avaliando a adsorção de elementos traços a solos: Sodré et. al. (2001); Nascimento et. al. (2004); Pierangelli et. al. (2005); Oliveira et. al.(2010); Tagliaferro et. al. (2011), Gonçalves et al (2013); Oliveira et. al. (2013); Rocha et al. (2013); Coelho et. al. (2014); Sodré et. al (2019); sendo que, na maioria dos casos, o modelo que melhor descreve o comportamento da adsorção é o de Freundlich, o que vai de encontro com o apresentado neste estudo. Porém, segundo Nascimento et al (2014), deve-se ressaltar a importância de que a equação de Freundlich é incapaz de prever dados de equilíbrio de adsorção quando são utilizadas faixas de concentrações extremamente elevadas.

Apesar de não possuir concentrações máximas no solo apresentadas em legislação, Rezende (2018) avaliando a qualidade do solo em áreas de disposição final de resíduos sólidos urbanos comparando área de disposição final antiga e atual e ainda vegetação nativa observou que na parte antiga os valores de lítio variam de 4,69 a 21,06 mg.kg-1 , com concentração média de 9,81 mg.kg-1 sendo, em alguns pontos amostrados a concentração maior do que os valores observados para a área de vegetação nativa, onde as concentrações variam de 4,22 a 5,47 $\mathrm{mg} \mathrm{kg}^{-1}$ e concentração média 4,83 $\mathrm{mg} \mathrm{kg}^{-1}$.

A Figura 2 apresenta as isotermas de adsorção de Li ao solo para diferentes tempos de contato, agrupando-se os resultados obtidos para a isoterma de Freundlich.

Figura 2. Isotermas de sorção Freundlich para os tempos de contato de 1, 12 e 24 horas.

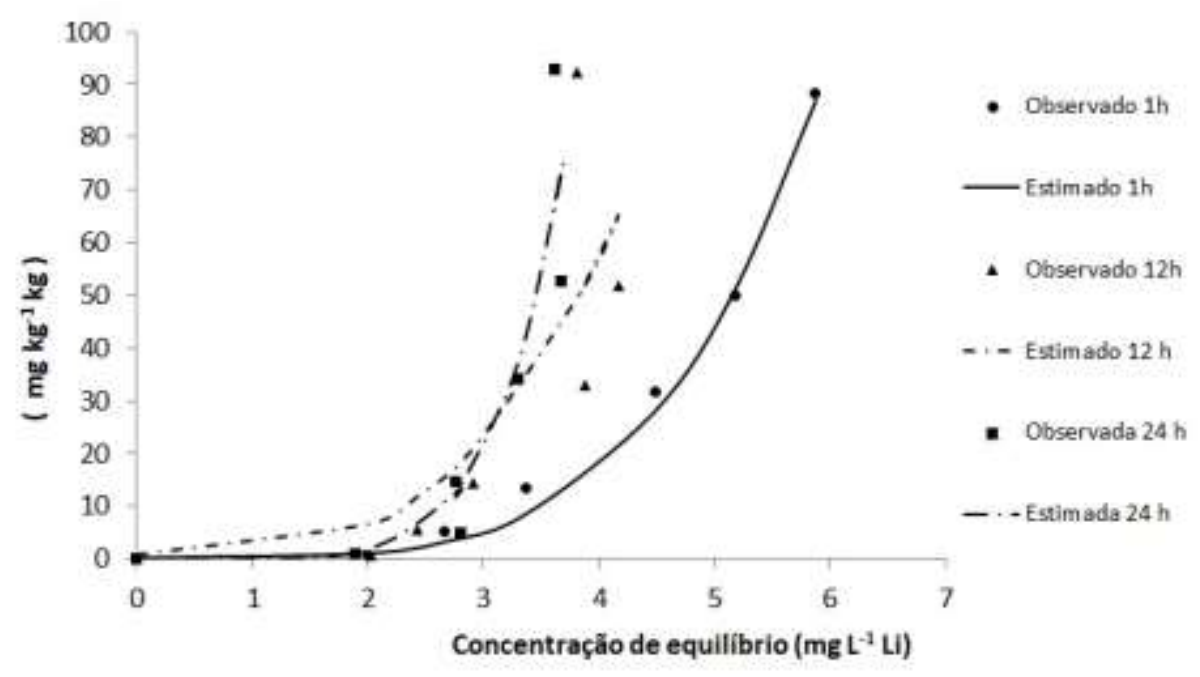

Fonte: Autores (2018).

Observou-se que a variação no tempo de contato provocou efeitos no processo de adsorção. Dentre os três tempos de contato e concentrações, as maiores sorções de lítio foram obtidas para o tempo de contato de 24 horas, porém observou-se comportamento semelhante para o tempo de contato de 12 horas, principalmente para as concentrações iniciais mais baixas. Já nas concentrações iniciais mais elevadas do adsorvato, como é o caso da isoterma de 24 horas, segundo Nascimento et. al 
(2014) a velocidade de adsorção tende a aumentar e atingem mais rapidamente a capacidade de adsorção, sendo proporcional à concentração do adsorvato.

Normas da USEPA (1992) sugere que o tempo de equilíbrio deve ser o mínimo necessário para estabelecer uma taxa de variação da concentração do soluto em solução, igual ou menor que 5\% por um intervalo de $24 \mathrm{~h}$, conforme relatado no estudo de Nicochellie et al. (2012), sendo que observou que o tempo de 6 horas foi insuficiente para atingir o equilíbrio. Santos et al (2006) estudou os tempos de contato de 1, 24, 48, e 72 horas na sorção do potássio em substrato caulinítico concluindo que o tempo de estabilização foi o de 24 horas. Diversos estudos na literatura comprovam ainda o tempo de contato de 24 horas nos estudos de adsorção em solos tropicais como os de Alleoni et al. (2005); Casagrande et al. (2004); Mellis et al. (2004); Soares (2004); e Vega et al. (2006).

\section{Conclusão}

O solo apresenta baixa capacidade de adsorção devido às suas características químicas, o que faz com que o poluente lixivie em maior quantidade para as camadas mais profundas, portanto, o solo pode ser mais vulnerável à contaminação das águas subterrâneas.

O modelo de Freundlich obteve o melhor ajuste para os dados observados, mostrando que há heterogenia da interação entre adsorvente e adsorvato, mesmo ocorrendo forte atração intermolecular entre Lítio e os componentes do solo.

Para os tempos avaliados, o maior tempo de contato entre o solo e o Lítio promoveu uma maior adsorção do último.

A partir dos estudos realizados com essa pesquisa, sugere-se trabalhos com maiores repetições e avaliação de outros íons metálicos como o alumínio e o manganês, além disso, estudos de deslocamento miscível e medição da condutividade hidráulica afim de verificar a mobilidade dos elementos e maior vulnerabilidade à contaminação das águas subterrâneas.

\section{Referências}

Alleoni, L. R. F., Cambri, M. A. \& Caires, E. F. (2005). Atributos químicos de um Latossolo de cerrado sob plantio direto, de acordo com doses e formas de aplicação de calcário. Revista Brasileira de Ciência do Solo, 29:923-934.

Baldotto, M. A. \& Velloso, A. C. X. (2014). Eletroquímica de solos modais e de sua matéria orgânica em ambientes tropicais. Revista Ceres, 61(6):1012-1021. http://dx.doi.org/10.1590/0034-737X201461060018.

Brasil. Ministério do Meio Ambiente. Conselho Nacional do Meio Ambiente-CONAMA. (2005). Resolução $n^{\circ} 357$, de 17 de março de 2005. Dispõe sobre a classificação dos corpos de água e diretrizes ambientais para o seu enquadramento, bem como estabelece as condições e padrões de lançamento de efluentes, e dá outras providências. Diário Oficial [da] República Federativa do Brasil, Poder Executivo, Brasília, DF. (53):58 - 63.

Brasil. (2015). Laudo Técnico Preliminar: Impactos ambientais decorrentes do desastre envolvendo o rompimento da barragem de Fundão, em Marina, Minas Gerais. In: Instituto Brasileiro de Meio ambiente - IBAMA. http://www.ibama.gov.br/phocadownload/noticias_ambientais/laudo_tecnico_preliminar. pdf. Acesso em: 21 de set. 2018.

Campos, M.C.C. (2010) Atributos dos solos e riscos de lixiviação de metais pesados em solos tropicais. Ambiência - Revista do Setor de Ciências Agrárias e Ambientais, 6 (3):547-565.

Casagrande, J. C., Jordão, C. B. Alleoni, L. R. F, \& Camargo, O. A. (2004). Copper desorption in a variable eletrical charge soil. Scientia Agricola, 61:196202.

Coelho, G. F, Gonçalves Junior, A. C., Tarley, C. R. T., Casarin, J. Nacke, H. \& Francziskowski A. (2014). Removal of metal ions Cd (II), Pb (II), and Cr (III) from water by the cashew nut shell Anacardium occidentale L. Ecological Engineering on Science. 73:514-525. https://doi.org/10.1007/s13201-018-0724-8

Cooney, D. O. (1999). Adsorption Design for Wastewater Treatment. CRC Press.

Corrêa, J. C., Büll, L. T., Paganini, W. S. \& Guerrini, I. A. (2008). Disponibilidade de metais pesados em latossolo com aplicação superficial de escória, lama cal, lodos de esgoto e calcário. Pesquisa Agropecuária Brasileira. 43(3):411-419. http://dx.doi.org/10.1590/S0100-204X2008000300017

Embrapa - Centro Nacional de Pesquisa de Solos (2011) Manual de métodos de análise de solos. (2a ed.), EMBRAPA, 230p.

Embrapa - Centro Nacional de Pesquisa de Solos. (2013). Sistema brasileiro de classificação de solos. (3a ed.), Embrapa. 353p.

Gonçalves, M. S, Bettin, J. P, Silva Junior, L. C. S., Sampaio, S. C., \& Dal Bosco, T. C. (2013). Adequação dos modelos de Langmuir e Freundlich na adsorção de cobre em solo argiloso do Sul do Brasil. Holos. 29(4):37-44. https://doi.org/10.15628/holos.2013.1458 
Lopes, Luciano M. N. (2016). O rompimento da barragem de Mariana e seus impactos socioambientais. Revista Sinapse Múltipla. 5(1):1-14. http://periodicos.pucminas.br/index.php/sinapsemultipla/article/view/11377/9677.

Mellis, E. V., Cruz, M. C. P. \& Casagrande, J. C. (2004). Nickel adsoption by soils in relation to pH, organic matter and iron oxides. Scientia Agricola.61:190195.

Mellis, E. V., \& Rodella, A. A. (2008). Influência do tempo de agitação na adsorção de Cd, Cu, Ni e Zn em Latossolo tratado com lodo de esgoto. Bragantia, Campinas. 67(4):977-982. http://dx.doi.org/10.1590/S0006-87052008000400021.

Nascimento, C. W. A., Barros, D. A. S. \& Melo, E. E. C. (2004). Soil chemical alterations and growth of maize and bean plants after sewage sludge application. Revista Brasileira de Ciência de Solo. 28(2):385-392. http://dx.doi.org/10.1590/S0100-06832004000200017

Nicochelli, L. M., Nascentes, R., Lima, E. B. N. R. \& Soares, F. S. C. (2012). Sorção de potássio em amostras de solo submetidas à aplicação de vinhaça. Revista Brasileira de Engenharia Agrícola e Ambiental. 16(7):754-760. http://dx.doi.org/10.1590/S1415-43662012000700008.

Oliveira, L. F. C., Nogueira, J. G., Frizzarim, S. S. Fia, R., Freitas, J. S. \& Fia, F, L, R. (2013). Sorção e mobilidade do lítio em solos de áreas de disposição final de resíduos sólidos urbanos. Engenharia Sanitária e Ambiental. 18(2):139-148. http://dx.doi.org/10.1590/S1413-41522013000200006.

Oliveira, L. F. C., Castro, M. L. L., Rodrigues, C. \& Borges, J. D. (2010). Isotermas de sorção de metais pesados em solos do cerrado de Goiás. Revista Brasileira de Engenharia Agrícola e Ambiental, 14(7):776-782. http://dx.doi.org/10.1590/S1415-43662010000700014.

Oliveira, L. F. C., Castro, M. L. L., Rodrigues, C. \& Borges, J. D. (2010a). Adsorção e deslocamento do íon cádmio em solos do cerrado. Revista Brasileira de Engenharia Agrícola e Ambiental.14(8):848-855. http://dx.doi.org/10.1590/S1415-43662010000800009.

Oliveira, L. F. C., Martinez, M. A., Pruski, F. F., Ruiz, H. A. \& Matos, A. T. (2000). Metodologia de amostragem de solo para a obtenção da condutividade hidráulica do solo saturado e da curva de eluição de solutos. Engenharia na Agricultura.8(2):112-121.

Pierangeli, M.A.P., Guilherme, L. R. G., Curi, N., Silva, M. L. N., De Lima, J. M. \& Costa, E.T.S. (2005). Effect of pH on cadmium adsorption and desorption in Brazilian Oxisols. Revista Brasileira de Ciência do Solo, Viçosa. 29(4,):361-367, 2005. http://dx.doi.org/10.1590/S0100-06832005000400005

Rezende, D. A. (2018). Qualidade do solo em local de disposição inadequada de resíduos sólidos em um município de pequeno porte. 81 f. Dissertação (Mestrado) Programa de Pós-graduação em Qualidade Ambiental. Universidade Federal de Uberlândia.

Rocha, P. R. R., Faria, A. T., Borges, L. G. F. C., Silva, L. O. C., Silva, A. A. \& Ferreira, E. (2013). A sorção e dessorção do diuron em quatro latossolos brasileiros. Planta Daninha, Viçosa-MG. 31(1):231-238. http://dx.doi.org/10.1590/S0100-83582013000100025.

Santos, L. V., Polivanov, H, Alamino, R. C. J., Silva \& V. H. G. (2006). Adsorção de cloreto e potássio em solos tropicais. Anuário do Instituto de Geociências, 29:101-121. http://www.anuario.igeo.ufrj.br/anuario_2006_2/anuario_2006_v29_2_101_121.pdf

Sobral, L. F. et al. (2015). Guia prático para interpretação de resultados de análises de solos. Embrapa Tabuleiros Costeiros Documentos (INFOTECA-E). https://ainfo.cnptia.embrapa.br/digital/bitstream/item/142260/1/Doc-206.pdf

Sodré, F. F., Lenzi, E. \& Costa, A. C. S. (2001). Utilização de Modelos Físicos-Químicos de Adsorção no Estudo do Comportamento do Cobre em solos Argilosos. Química Nova, Maringá, 24(3):324-330, 2001. http://dx.doi.org/10.1590/S0100-40422001000300008.

Sodré, F. F. Lima, L. C. C., Busato \& J. G. (2019). Copper and lead adsorption as influenced by organic matter in soils from a tropical toposequence with different chemical and mineralogical attributes. Journal Archives of Agronomy and Soil Science, 65(3):334 -344. https://doi.org/10.1080/03650340.2018.1503413

Sposito, G. (1989) The chemistry of soils. Oxford University Press. 234p.

Tagliaferro, G. V., Pereira, P. H. F., Rodrigues, L. A. \& Silva, M. L.C. P. (2011). Adsorção de chumbo, cádmio e prata em óxido de nióbio hidratado preparado pelo método da precipitação em solução homogênea. Química Nova, 34(1):101-105. http://dx.doi.org/10.1590/S0100-40422011000100020.

Usepa - United States Environmental Protection Agency. (1992). Batch - type procedures for estimating soil adsorption of chemicals. EPA/530/SW-87/006-F., $101 \mathrm{p}$

Van Genuchten, M. T. \& Wierenga, P. J. (1986). Solute dispersion coefficients and retardation factors. In: Klute, A. Methods of soil analysis: part 1 - physical and mineralogical methods. Madison: Soil Science Society of America, 1025-1054.

Vega, F. A., Covelo, E. F. \& Andrade, M. L. (2006). Competitive sorption and desorption of heavy metals in mine soils: Influence of mine soil characteristics. Journal of Colloid and Interface Science, 298:582-592.

Vilela, N. M. S., Thebaldi, M. S., Leal, B. P., Silva, A. V. \& Martins, I. P. (2018). Transport parameters of potassium from different sources in soil columns. Engenharia Agrícola, 38(1):135-141, 2018. http://dx.doi.org/10.1590/1809-4430-eng.agric.v38n1p135-141/2018

Yamamura, A. P. G. (2009). Aplicação de nanotecnologia no meio ambiente: Biossorvente magnético na remoção de urânio. Dissertação (Mestrado em Tecnologia Nuclear - Materiais) - Instituto de Pesquisas Energéticas e Nucleares, Universidade de São Paulo, São Paulo.

Zanella, L. C. H. (2013). Metodologia de pesquisa. Florianópolis: Departamento de Ciências da Administração/UFSC, 134 p. 